\title{
A case series on the potential effect of omega-3-fatty acid supplementation on 24-h heart rate variability and its circadian variation in children with attention deficit (hyperactivity) disorder
}

\author{
Reiner Buchhorn ${ }^{1} \cdot$ Julian Koenig $^{2} \cdot$ Marc N. Jarczok $^{3} \cdot$ Hanna Eichholz $^{1}$. \\ Christian Willaschek $^{1} \cdot$ Julian F. Thayer $^{4} \cdot$ Michael Kaess $^{2,5}$
}

Received: 17 March 2017 / Accepted: 25 September 2017 / Published online: 3 October 2017

(c) Springer-Verlag GmbH Austria 2017

\begin{abstract}
Attention deficit disorder with and without hyperactivity (ADHD) in children is associated with decreased 24-h heart rate variability (HRV). Previous research has shown that supplementation of omega-3-fatty acid increases HRV. Here, we aimed to investigate whether the supplementation of omega-3-fatty acids would increase 24-h HRV in an uncontrolled case series of children with ADHD. HRV was recorded in 18 children and adolescents (age $13.35 \pm 2.8$ years) before and after omega-3 supplementation. Preliminary results indicate that omega- 3 supplementation in children with $\mathrm{AD}(\mathrm{H}) \mathrm{D}$ may reduce mean heart rate and increase its variability. Future studies would do well to implement randomized, placebo-controlled designs with greater methodological rigor.
\end{abstract}

Reiner Buchhorn and Julian Koenig have contributed equally to this work.

Reiner Buchhorn

reiner.buchhorn@ckbm.de

1 Department of Paediatrics, Klinik für Kinder- und Jugendmedizin, Caritas Krankenhaus, Uhlandstr. 7, 97980 Bad Mergentheim, Germany

2 Section for Translational Psychobiology in Child and Adolescents Psychiatry, Department of Child and Adolescent Psychiatry, Centre for Psychosocial Medicine, Heidelberg University, Heidelberg, Germany

3 Institute of Medical Psychology, Center for Psychosocial Medicine, Heidelberg University, Heidelberg, Germany

4 Department of Psychology, The Ohio State University, Columbus, OH, USA

5 Clinic for Child and Adolescent Psychiatry, University Hospital Heidelberg, Heidelberg, Germany
Keywords Heart rate variability $\cdot$ ADHD $\cdot$ Vagal activity $\cdot$ Circadian variation $\cdot$ Omega-3

\section{Introduction}

It has previously been shown that untreated children with attention deficit disorder with and without hyperactivity $[\mathrm{AD}(\mathrm{H}) \mathrm{D}]$ exhibit decreased 24-h heart rate variability [HRV] assessed via Holter ECGs (Buchhorn et al. 2012), albeit absence of altered HRV in short-term resting state recordings (Koenig et al. 2017). Autonomic imbalance, reflected by decreased HRV, is an important indicator of an enhanced cardiovascular morbidity and mortality risk in adults (Wulsin et al. 2015) as well as children and adolescents (Farah et al. 2014; Vrijkotte et al. 2015). Ongoing debate in the $\mathrm{AD}(\mathrm{H}) \mathrm{D}$ literature predominantly focuses on the proarrhythmogenic side effects of psycho-stimulants (Triedman and Alexander 2010) and not on the well-known enhanced cardiovascular risk in patients with psychiatric disease (Neylon et al. 2013).

Nutritional intake of omega-3-fatty acids has a potential positive impact on cognition (Cooper et al. 2015) and emotional dysregulation (Cooper et al. 2016; Messamore and McNamara 2016) associated with $\mathrm{AD}(\mathrm{H}) \mathrm{D}$, although the existing evidence is mixed (Chang et al. 2017). The DOLAB study showed low blood long-chain omega-3 fatty acids in children with poor cognitive performance and behavior (Montgomery et al. 2013). Several prospective randomized controlled trials support the supplementation of omega-3fatty acids in children with $\mathrm{AD}(\mathrm{H}) \mathrm{D}$ to improve cognition (Gow et al. 2015; Widenhorn-Müller et al. 2014). Beneficial effects on inattention in $\mathrm{AD}(\mathrm{H}) \mathrm{D}$ and healthy children (Bos et al. 2015) and other psychopathologies across the lifespan have also been reported (Sinn et al. 2010, 2012). 
However, other studies showed that omega-3-fatty acid supplementation effects cognition only in patients with reduced omega-3 levels (Hawkey and Nigg 2014). Further, omega-3 fatty acids might improve cardiovascular function in children with $\mathrm{AD}(\mathrm{H}) \mathrm{D}$ and thereby reduce the associated cardiovascular risk, but more research is needed on this topic (Simopoulos 2008).

Previous research has shown that the supplementation of omega-3-fatty acid increases HRV (Carney et al. 2010; Christensen 2011; O'Keefe et al. 2006; Romieu et al. 2005). Here, drawing on routine clinical data from a pediatric cardiology department, we aimed to illustrate potential effects of omega-3-fatty acid supplementation on 24-h HRV in children with $\mathrm{AD}(\mathrm{H}) \mathrm{D}$, based on a case series. We assumed that children with $\mathrm{AD}(\mathrm{H}) \mathrm{D}$ would show increased 24-h HRV and reduced heart rate (HR) after omega-3-fatty acids supplementation.

\section{Methods}

\section{Patients and procedures}

A total of 18 cases, children and adolescents (age $13.35 \pm 2.8$ years; size $156.8 \pm 16.4 \mathrm{~cm}$; weight

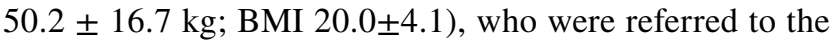
outpatient clinic for pediatrics of the Caritas Hospital Bad Mergentheim between the years 2010-2016 were identified based on clinical files. Patients with a diagnosis of $\mathrm{AD}(\mathrm{H})$ $\mathrm{D}$, made by an external child psychiatrist according ICD-10 diagnostic criteria, were referred to the pediatric cardiology department for evaluation. All patients and their parents were informed about the multimodal therapy for $\mathrm{AD}(\mathrm{H}) \mathrm{D}$ (medications and behavioral therapy) and the possibility to supplement with omega-3-fatty acids. Of the cases included for analysis, some used omega-3 prior to stimulant treatment $(n=4)$; some after termination of stimulant treatment because of the occurrence of side effects $(n=4)$; and the majority used omega- 3 to reduce the cardiovascular risk during stimulant treatment $(n=10)$. If the supplementation was not covered by health insurance, patients usually purchased different products based upon 1-2 $\mathrm{g}$ fish oil per day from discounters.

\section{Hour ECG and analysis of heart rate variability}

Two 24-h Holter ECGs were recorded. One before supplementation with omega-3-fatty acids and a second one after 143.7 days on average (range 42-490 days). Given the large variance in days between assessments due to the naturalistic design of the study, potential outcome effects were addressed in later analysis. A two-channel Holter monitor (Pathfinder,
Spacelabs, Germany) was used for recording, while the children followed their normal daily routines. All Holter recordings were reviewed by an experienced cardiologist and were edited to validate the system's QRS labeling. Measures of HRV were calculated using inter-beat intervals between adjacent R-peaks. The Holter ECGs were analyzed as average values from the entire $24 \mathrm{~h}$ of analyzable data and additionally for circadian rhythm analysis as mean hourly values with regard to the time of day. Measurement and interpretation of HRV parameters in the current sample were standardized according to the Task Force Guidelines (Task Force of the European Society of Cardiology and the North American Society of Pacing and Electrophysiology 1996). A minimum of $23 \mathrm{~h}$ of analyzable data and minimally $95 \%$ of analyzable $\mathrm{RR}$ intervals were required for the data to be included. The presentation focuses on three parameters, ${ }^{1}$ including heart rate (mean RR intervals) and one time-domain (the square root of the mean of the sum of squared differences between adjacent RR intervals (RMSSD)) and one frequency-domain (high-frequency HRV via fast Fourier transformation over the frequency of $0.15-0.4 \mathrm{~Hz}$ ) measure of vagally mediated HRV.

\section{Statistical analysis}

To investigate changes in 24-h HRV recordings taken preand post-omega-3 supplementation, a threefold approach was chosen. First, the overall 24-h means of all indices were compared using paired $t$ test for dependent samples. Second, mixed linear regression analyses were used to investigate main effects of supplementation (baseline versus omega-3) over repeated measures (main effect: hourly means) on all indices. To adjust for the time difference between repeated measures, we further tested such effect but found no significant influence of the variance in days between assessments. Third, we used a cosine function analysis approach (trigonometric linear regression) to model differences in circadian variation patterns and graphically display them. In a first step, three individual-level cosine function parameters were estimated for each individual 24-h time series to quantify the following circadian variation pattern (CVP) parameters: MESOR (the rhythm adjusted 24-h mean level), amplitude (the distance between MESOR and the highest maximum value of the cosine curve), and acrophase (the phase shift of amplitude from a given reference time point when the highest oscillation is reached). In a second step, these parameters were subjected to mixed linear regression

\footnotetext{
1 The selection of parameters was limited during the review process. Upon request, the authors provide additional data on the standard deviation of RR intervals (SDNN), the percentage of pairs of adjacent $\mathrm{RR}$ intervals differing by more than $50 \mathrm{~ms}$ (pNN50), very low frequency (VLF, <.04 Hz), and low frequency (LF, .04-.15 Hz) HRV.
} 
Table 1 Means of HRV indices at baseline and after omega-3 supplementation

\begin{tabular}{|c|c|c|c|c|c|c|c|c|c|}
\hline & \multicolumn{3}{|l|}{$24 \mathrm{~h}$} & \multicolumn{3}{|l|}{ Day } & \multicolumn{3}{|l|}{ Night } \\
\hline & Baseline & Omega-3 & $p$ & Baseline & Omega-3 & $p$ & Baseline & Omega-3 & $p$ \\
\hline Mean RR & $667.24(68.59)$ & $726.82(73.81)$ & .001 & $589.39(63.11)$ & $639.88(83.93)$ & .003 & $744.12(96.80)$ & $811.79(85.91)$ & .006 \\
\hline RMSSD & $34.488(12.47)$ & $45.06(22.21)$ & .017 & $25.47(8.61)$ & $34.04(17.02)$ & .017 & $43.40(18.65)$ & $55.74(28.44)$ & .021 \\
\hline $\mathrm{HF}$ & $472.00(264.65)$ & $623.83(341.70)$ & .010 & $282.47(163.91)$ & $429.49(257.40)$ & .005 & $659.06(399.10)$ & $813.02(468.75)$ & .046 \\
\hline
\end{tabular}

Values are means and standard deviations in brackets for 24-h recordings; daytime recordings (6:00 am-6:00 pm) and nighttime recordings (6:00 pm-6:00 am); all observations $n=18 ; p$ values refer to paired $t$ test (two-sided)

Mean $R R$ mean RR interval, $R M S S D$ square root of the mean of the sum of squares of differences between adjacent RR intervals, $H F$ highfrequency power $(0.15-0.4 \mathrm{~Hz})$
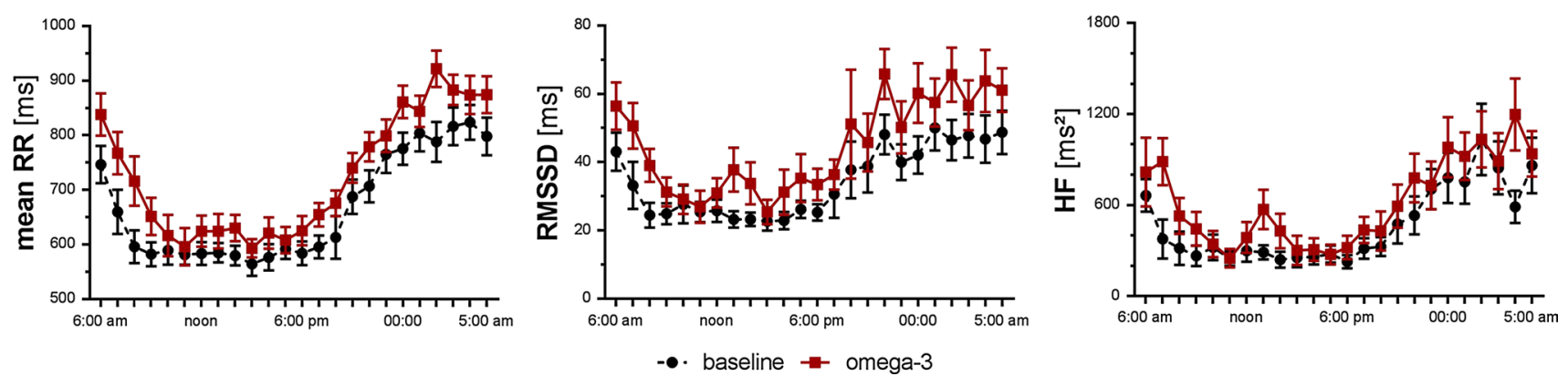

Fig. 1 Change in heart rate and heart rate variability based on hourly means from baseline to omega-3 supplementation; mean $R R$ mean RR interval, $R M S S D$ square root of the mean of the sum of squares of differences between adjacent RR intervals, $H F$ high frequency $(0.15-0.4 \mathrm{~Hz})$

models to estimate the impact of omega-3 supplementation on MESOR, amplitude, and acrophase. Accordingly, time (baseline versus omega-3) differences were graphically illustrated using predicted values and the corresponding $95 \% \mathrm{CI}$ of the standard errors of the prediction. All analyses were performed using Stata/SE (Version 14.1; StataCorp LP, College Station, TX, US) with alpha set to .05. Graphs were prepared using GraphPad Prism (version 6.0, GraphPad Software Inc., USA).

\section{Results}

Descriptive statistics on 24-h as well as day- and nighttime means of the different indices by the time of measurement are provided in Table 1 . In mixed linear regression analysis on repeated measures of hourly means, omega-3 supplementation had a significant main effect on mean RR $\left(\chi_{(1)}^{2}=17.11, p<.001\right), \operatorname{RMSSD}\left(\chi_{(1)}^{2}=7.23, p=.0072\right)$, and $\operatorname{HF}\left(\chi_{(1)}^{2}=9.29, p=.0023\right)$. The time of day had a significant main effect on all dependent variables, including mean RR $\left(\chi_{(17)}^{2}=818.21, p<.001\right)$, RMSSD $\left(\chi_{(17)}^{2}=395.87\right.$, $p<.001)$, and HF $\left(\chi_{(17)}^{2}=175.06, p<.001\right)$. Analysis revealed significant interactions of omega-3 supplementation and the time of day on mean RR $\left(\chi_{(17)}^{2}=118.39\right.$, $p<.001)$, RMSSD $\left(\chi_{(17)}^{2}=651.34, p<.001\right)$, and HF $\left(\chi_{(17)}^{2}=55.08, p<.001\right)$. Findings are illustrated in Fig. 1. Changes in CPV from baseline to post-omega-3 supplementation are illustrated in Fig. 2. In mixed linear regression analysis on CVP parameters of HRV, omega-3 supplementation had a significant effect on the MESOR of mean RR $\left(\chi_{(1)}^{2}=16.03, p<.001\right), \operatorname{RMSSD}\left(\chi_{(1)}^{2}=6.92, p=.009\right)$, and $\operatorname{HF}\left(\chi_{(1)}^{2}=8.16, p=.004\right)$. Omega-3 supplementation had a significant effect on the amplitude of mean RR $\left(\chi_{(1)}^{2}=4.92\right.$, $p=.027)$ and $\operatorname{RMSSD}\left(\chi_{(1)}^{2}=4.62, p=.032\right)$, but not HF $\left(\chi_{(1)}^{2}=1.65, p=.200\right)$. Omega-3 supplementation had no significant effect on any of the acrophase parameters.

\section{Discussion}

The present case series, drawing on routine clinical data, aimed to elucidate potential effects of omega-3 supplementation on long-term recordings of HRV in children with $\mathrm{AD}(\mathrm{H}) \mathrm{D}$. While the study has major methodological shortcoming, this case series indicates that omega-3 supplementation in children with $\mathrm{AD}(\mathrm{H}) \mathrm{D}$ may reduce mean heart rate and increase its variability on a variety of time- and frequency-domain measures. Analysis or recordings collected before and after omega-3 supplementation revealed changes in measures of the overall mean HRV and measure of amplitude in mean RR and RMSSD. 

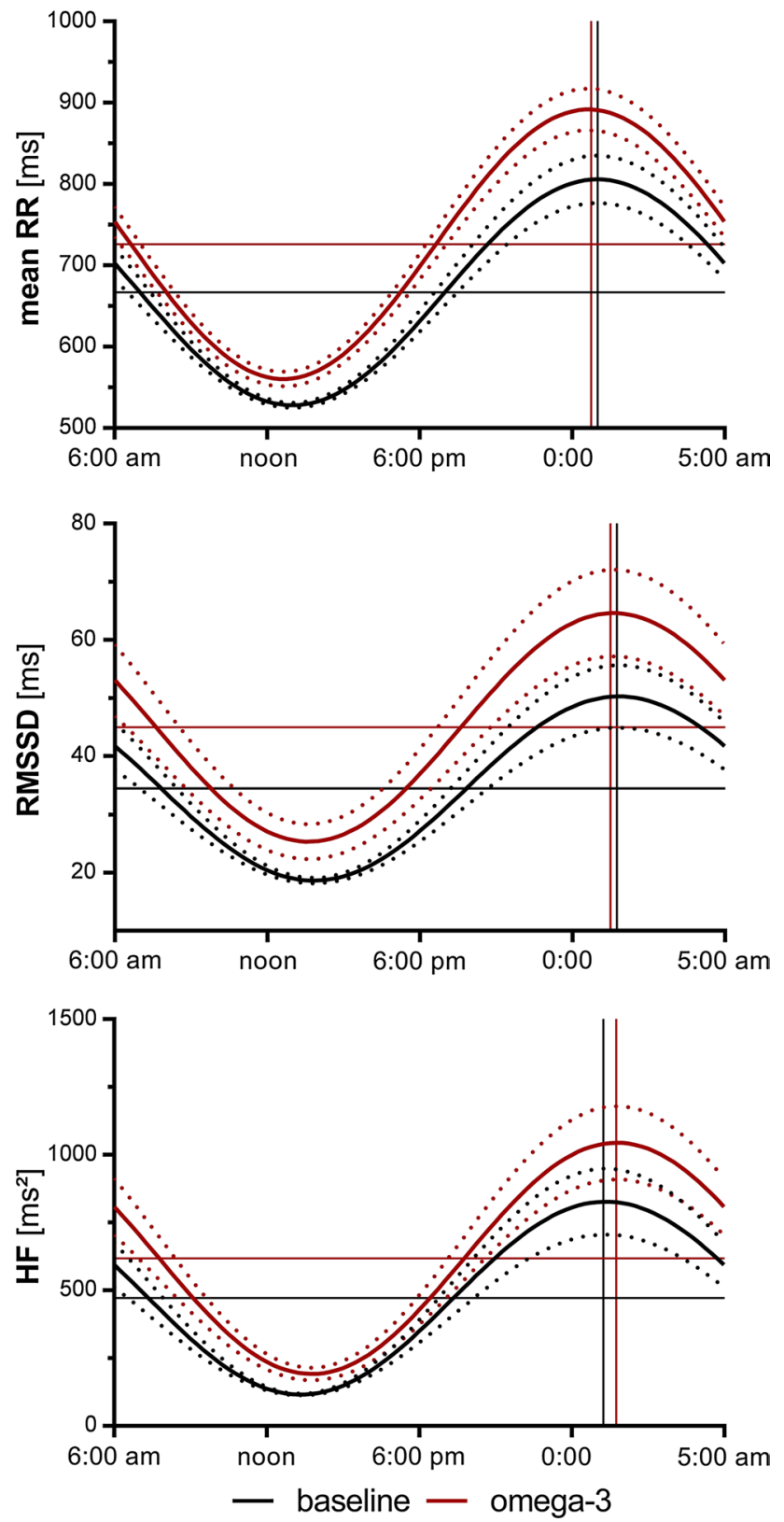

Fig. 2 Change in heart rate and heart rate variability based on circadian variation pattern from baseline to omega-3 supplementation; mean $R R$ mean RR interval, $R M S S D$ square root of the mean of the sum of squares of differences between adjacent RR intervals, $H F$ high-frequency power $(0.15-0.4 \mathrm{~Hz})$; dotted lines indicate the $95 \%$ confidence interval

Reduced HRV is an important marker of enhanced cardiovascular risk (Thayer et al. 2010). Only a few studies in children with $\mathrm{AD}(\mathrm{H}) \mathrm{D}$ to date have included cardiovascular endpoints. The majority of the existing studies addressed the association of cardiovascular adverse events and stimulant treatment (Dalsgaard et al. 2014). Stimulants have been reported to increase resting heart rate and elevate systolic blood pressure compared to placebo in adults with $\mathrm{AD}(\mathrm{H}) \mathrm{D}$ (Mick et al. 2013).

However, the present case series has several limitations that need to be addressed in future research. First, we studied a relatively small sample of children and adolescents from a naturalistic clinical sample using routine data, referred to a pediatric cardiology department, who were not characterized in detail. In particular, a lack of standardized psychiatric assessment and information about child and adolescent psychiatric and psychotherapeutic treatment limit the generalizability of the findings, as other treatments may underlie the increase in HRV. This includes a lack of standardized reporting of $\mathrm{AD}(\mathrm{H}) \mathrm{D}$ symptom severity before and after omega-3 supplementation. Further, the supplementation of omega-3 fatty acids was not standardized by any means (i.e., exact compound, dose, duration of supplementation).

We were not able to gain information on the EPA/DHA content and dose of the used brands of supplements and therefore are not able to determine whether appropriate doses of EPA/DHA have been taken and whether the ratio between EPA and DHA was appropriate. Further, we cannot rule out potential effects of combined stimulant treatment, as some patients used omega-3 prior to stimulant treatment, some after termination of stimulant treatment, and some during stimulant treatment. Future studies would do well to implement randomized, placebo-controlled designs and adhere to a strict protocol with respect to the measurement point of HRV, as we cannot rule out any other factors (i.e., changes in lifestyle habits such as physical activity) potentially influencing HRV. Further, we did not measure red blood cell PUFA levels, prohibiting any conclusion with respect to the compliance with omega-3 supplementation, or a potential dose-response relation between omega-3 intake and changes in HR or HRV. Albeit these methodological limitations and shortcomings, the case series illustrates preliminary empirical data, illustrating that HRV may be an interesting biological marker that may be used to assess effects of omega-3 supplementation in ADHD. Longitudinal trials assessing the potential of omega-3-fatty acid supplementation to improve the risk of arrhythmias (London et al. 2007) and hypertension (Buchhorn and Christian 2014) in children with $\mathrm{AD}(\mathrm{H}) \mathrm{D}$ are warranted.

Acknowledgements JK and MNJ are supported by a PhysicianScientist-Fellowship provided by the Medical Faculty of Heidelberg University.

\section{Compliance with ethical standards}

Conflict of interest The authors have no conflict of interest, real or perceived, to declare. 


\section{References}

Bos DJ, Oranje B, Veerhoek ES, Van Diepen RM, Weusten JM, Demmelmair $\mathrm{H}$ et al (2015) Reduced symptoms of inattention after dietary omega-3 fatty acid supplementation in boys with and without attention deficit/hyperactivity disorder. Neuropsychopharmacol Off Publ Am Coll Neuropsychopharmacol 40:2298-2306

Buchhorn R, Christian W (2014) Ventricular arrhythmias in children with attention deficit disorder-a symptom of autonomic imbalance? Cardiol Young 24:120-125

Buchhorn R, Conzelmann A, Willaschek C, Störk D, Taurines R, Renner TJ (2012) Heart rate variability and methylphenidate in children with ADHD. Atten Deficit Hyperact Disord 4:85-91

Carney RM, Freedland KE, Stein PK, Steinmeyer BC, Harris WS, Rubin EH et al (2010) Effect of omega-3 fatty acids on heart rate variability in depressed patients with coronary heart disease. Psychosom Med 72:748-754

Chang J-C, Su K-P, Mondelli V, Pariante CM (2017) Omega-3 polyunsaturated fatty acids in youths with attention deficit hyperactivity disorder (ADHD): a systematic review and meta-analysis of clinical trials and biological studies. Neuropsychopharmacol Off Publ Am Coll Neuropsychopharmacol. doi:10.1038/npp.2017.160

Christensen JH (2011) Omega-3 polyunsaturated fatty acids and heart rate variability. Front Physiol. doi:10.3389/fphys.2011.00084

Cooper RE, Tye C, Kuntsi J, Vassos E, Asherson P (2015) Omega-3 polyunsaturated fatty acid supplementation and cognition: a systematic review and meta-analysis. J Psychopharmacol 29:753-763

Cooper RE, Tye C, Kuntsi J, Vassos E, Asherson P (2016) The effect of omega-3 polyunsaturated fatty acid supplementation on emotional dysregulation, oppositional behaviour and conduct problems in ADHD: a systematic review and meta-analysis. J Affect Disord 190:474-482

Dalsgaard S, Kvist AP, Leckman JF, Nielsen HS, Simonsen M (2014) Cardiovascular safety of stimulants in children with attentiondeficit/hyperactivity disorder: a nationwide prospective cohort study. J Child Adolesc Psychopharmacol 24:302-310

Farah BQ, Barros MVG, Balagopal B, Ritti-Dias RM (2014) Heart rate variability and cardiovascular risk factors in adolescent boys. J Pediatr 165:945-950

Gow RV, Hibbeln JR, Parletta N (2015) Current evidence and future directions for research with omega-3 fatty acids and attention deficit hyperactivity disorder. Curr Opin Clin Nutr Metab Care 18:133-138

Hawkey E, Nigg JT (2014) Omega-3 fatty acid and ADHD: blood level analysis and meta-analytic extension of supplementation trials. Clin Psychol Rev 34:496-505

Koenig J, Rash JA, Kemp AH, Buchhorn R, Thayer JF, Kaess M (2017) Resting state vagal tone in attention deficit (hyperactivity) disorder: a meta-analysis. World J Biol Psychiatry Off J World Fed Soc Biol Psychiatry 18:256-267

London B, Albert C, Anderson ME, Giles WR, Wagoner DRV, Balk E et al (2007) Omega-3 fatty acids and cardiac arrhythmias: prior studies and recommendations for future research. Circulation 116:e320-e335

Messamore E, McNamara RK (2016) Detection and treatment of omega-3 fatty acid deficiency in psychiatric practice: rationale and implementation. Lipids Health Dis. doi:10.1186/ s12944-016-0196-5
Mick E, McManus DD, Goldberg RJ (2013) Meta-analysis of increased heart rate and blood pressure associated with CNS stimulant treatment of ADHD in adults. Eur Neuropsychopharmacol J Eur Coll Neuropsychopharmacol 23:534-541

Montgomery P, Burton JR, Sewell RP, Spreckelsen TF, Richardson AJ (2013) Low blood long chain omega-3 fatty acids in UK children are associated with poor cognitive performance and behavior: a cross-sectional analysis from the DOLAB study. PLoS ONE 8:e66697

Neylon A, Canniffe C, Anand S, Kreatsoulas C, Blake GJ, Sugrue D, McGorrian C (2013) A global perspective on psychosocial risk factors for cardiovascular disease. Prog Cardiovasc Dis 55:574-581

O'Keefe JH Jr, Abuissa H, Sastre A, Steinhaus DM, Harris WS (2006) Effects of omega-3 fatty acids on resting heart rate, heart rate recovery after exercise, and heart rate variability in men with healed myocardial infarctions and depressed ejection fractions. Am J Cardiol 97:1127-1130

Romieu I, Téllez-Rojo MM, Lazo M, Manzano-Patiño A, Cortez-Lugo M, Julien P et al (2005) Omega-3 fatty acid prevents heart rate variability reductions associated with particulate matter. Am J Respir Crit Care Med 172:1534-1540

Simopoulos AP (2008) The importance of the omega-6/omega-3 fatty acid ratio in cardiovascular disease and other chronic diseases. Exp Biol Med Maywood NJ 233:674-688

Sinn N, Milte C, Howe PRC (2010) Oiling the brain: a review of randomized controlled trials of omega- 3 fatty acids in psychopathology across the lifespan. Nutrients 2:128-170

Sinn N, Milte CM, Street SJ, Buckley JD, Coates AM, Petkov J, Howe PRC (2012) Effects of n-3 fatty acids, EPA v. DHA, on depressive symptoms, quality of life, memory and executive function in older adults with mild cognitive impairment: a 6-month randomised controlled trial. Br J Nutr 107:1682-1693

Task Force of the European Society of Cardiology and the North American Society of Pacing and Electrophysiology (1996) Heart rate variability: standards of measurement, physiological interpretation and clinical use. Task Force of the European Society of Cardiology and the North American Society of Pacing and Electrophysiology. Circulation 93:1043-1065

Thayer JF, Yamamoto SS, Brosschot JF (2010) The relationship of autonomic imbalance, heart rate variability and cardiovascular disease risk factors. Int J Cardiol 141:122-131

Triedman JK, Alexander ME (2010) Needle in a haystack. Circulation 121:1283-1285

Vrijkotte TGM, van den Born B-JH, Hoekstra CMCA, Gademan MGJ, van Eijsden M, de Rooij SR, Twickler MTB (2015) Cardiac autonomic nervous system activation and metabolic profile in young children: the ABCD study. PLoS ONE 10:e0138302

Widenhorn-Müller K, Schwanda S, Scholz E, Spitzer M, Bode H (2014) Effect of supplementation with long-chain $\omega$-3 polyunsaturated fatty acids on behavior and cognition in children with attention deficit/hyperactivity disorder (ADHD): a randomized placebo-controlled intervention trial. Prostaglandins Leukot Essent Fatty Acids 91:49-60

Wulsin LR, Horn PS, Perry JL, Massaro J, D’Agostino R (2015): Autonomic imbalance as a predictor of metabolic risks, cardiovascular disease, diabetes, and mortality autonomic imbalance predicts CVD, DM, mortality. J Clin Endocrinol Metab. jc20144123 